\title{
Common peroneal nerve palsy after TKA in valgus deformities; a systematic review
}

\author{
Raymond Puijk ${ }^{1 *} \mathbb{D}$, Rachid Rassir ${ }^{1}$, Laura M. Kok ${ }^{1}$, Inger N. Sierevelt ${ }^{1,2}$ and Peter A. Nolte ${ }^{1}$
}

\begin{abstract}
Purpose: The aim of this systematic review is to investigate the prevalence of Common Peroneal Nerve Palsy after total knee arthroplasty in valgus deformities. Furthermore, the effectiveness of a peroneal nerve release prior to arthroplasty to prevent the palsy will be investigated.

Methods: PubMed and Google Scholar were searched. Search terms regarding valgus deformity and total knee arthroplasty were used. Data analysis and extraction were performed using the web application 'Rayyan QCRI' according to PRISMA guidelines and screened according to the inclusion and exclusion criteria.
\end{abstract}

Results: Twenty-seven studies were included, representing 1397 valgus knees. Knee balancing was performed in 19 studies with lateral soft tissue releases (1164 knees) and 8 studies (233 knees) with an additional osteotomy. Two studies (41 knees) in the lateral soft tissue release group conducted a peroneal nerve release simultaneous to arthroplasty. Common peroneal nerve palsies occurred in 26 cases (1.9\%). Overall, no significant difference in palsy ratio between studies was found by using a peroneal nerve release $(p=0.90)$, between lateral soft tissue releases and osteotomies $(p=0.11)$ or between releases of specific ligaments.

Conclusion: Common peroneal nerve palsies occur in 1.9\% of the cases after total knee arthroplasty in valgus deformities. No difference in the number of palsies was seen when using a peroneal nerve release or using different balancing techniques. However, literature about peroneal nerve releases was very limited, therefore, the effectiveness of a peroneal nerve release remains unclear.

Level of evidence: LEVEL III: Systematic review.

Keywords: Valgus deformity, Total knee arthroplasty, Soft tissue release, Peroneal nerve release, Common peroneal nerve palsy

\section{Introduction}

Common peroneal nerve palsy (CPNP) is a feared complication after total knee arthroplasty (TKA). Previous studies show valgus deformity and flexion contracture as predisposing factors to develop CPNP [1-5]. An increased anatomical femorotibial angle (aFTA) of $>10^{\circ}$ is commonly used to define a valgus knee [6-8]. In the literature, the reported CPNP incidence after TKA in

\footnotetext{
*Correspondence: rpuijk@spaarnegasthuis.nl

1 Department of Orthopaedic Surgery, Spaarne Gasthuis, Spaarnepoort 1, 2130AT, Hoofddorp, the Netherlands

Full list of author information is available at the end of the article
}

valgus deformities with an aFTA $>10^{\circ}$ (TKA-V) ranges between $0.3-9.5 \%$ [3, 9-17]. Injury of the common peroneal nerve $(\mathrm{CPN})$ can be caused by indirect damage due to stretch or ischaemia after correction, or by direct injury due to laceration of the CPN during lateral soft tissue release (STR) $[8,11,12,18-20]$. Commonly contracted and released ligaments in valgus knees include the iliotibial band (ITB), posterolateral capsule (PLC), lateral collateral ligament (LCL), popliteus tendon (POP) and lateral gastrocnemius tendon $[8,21]$. The selection of ligaments to be released depend mainly on the tightness of the ligaments in extension and/or flexion. Ligaments can be released through pie-crusting [7, 13, 20, 22-25], 
a subperiosteal release $[13,23,26-28]$ or in a transverse manner. Also, shifting the insertion of a ligament by use of an osteotomy (OT), like a lateral femoral epicondyle osteotomy (LFEO) [29-37] or medial femoral epicondyle osteotomy (MFEO) [30, 33] is used. However, an overzealous release may result in direct injury of the $\mathrm{CPN}$, late-onset instability and even a higher revision rate [33, $38,39]$.

Recovery from CPNP usually take place within a year; however, residual damage is certainly not uncommon [1, $4,5,12]$. As CPNP has serious consequences, orthopaedic surgeons aim to prevent this complication by a concomitant peroneal nerve release (PNR) $[8,40]$. A PNR is a procedure performed simultaneously to TKA-V, which explores the nerve and removes the constricted dressings to release the CPN. Therefore, it yields the nerve to have more capacity to extend and protects it against mechanical stretching after balancing the knee properly during TKA-V. Due to the limited number of studies investigating PNR, no consensus has yet been reached on the value and indication of the procedure.

This systematic review primarily attempts to investigate the CPNP incidence after TKA-V and the rate will be compared between different valgus correcting techniques, including lateral STR and OT. Secondarily, the effectiveness of a PNR in preventing CPNP after TKA-V will be investigated.

\section{Material and methods}

\section{Search strategy}

A librarian-assisted comprehensive search of the literature was performed in October 2020 in PubMed and Google Scholar. The primary search was mainly focused on the surgical treatment and outcome of TKA-V. Search terms and associated synonyms that were included in the search are displayed in Table 1. A total of 3.945 articles were identified through PubMed and Google Scholar (Fig. 1 [Additional file 1]). The analysis was done according to the 'Preferred Reporting Items of Systematic review and Meta-analyses' (PRISMA) [41]. Through the web application 'Rayyan QCRI' [42], duplicates were removed and the remaining articles were screened for eligibility, according to the screening criteria (Table 2). The screening was independently done by three reviewers (X, $\mathrm{X}$ and $\mathrm{X}$ ), a fourth reviewer (X) was consulted in case of doubt about the suitability of an article [43]. To ensure no relevant articles were omitted, a cross-reference check was performed on the included articles. A consensus was achieved on all included articles based on inclusion and exclusion criteria.

\section{Data extraction}

Data were extracted from each study by the first author (X) and collected in Microsoft Excel 2020 (Microsoft Corp., Redmond, WA, USA). Data of each article that was collected, included first author, study design (pro- or retrospective), study characteristics (year of publication, country, number of knees and patients), patient characteristics (age, gender, body mass index [12], the ratio of osteoarthritis (OA) or rheumatoid arthritis and followup), inclusion and exclusion criteria, alignment details (classification type, pre-and postoperative mechanical axis, anatomical axis (aFTA), range of motion and flexion contracture angle), peroneal nerve release, arthroplasty (surgical approach, implant design), lateral soft tissue release (technique, sequence and affected ligaments),

Table 1 PubMed search strategy, October 2020

(((()(((“arthroplasty, replacement, knee"[MeSH Terms] OR (("arthroplasty"[All Fields] AND “replacement"[All Fields]) AND “knee"[All Fields])) OR "knee replacement arthroplasty"[All Fields]) OR (("total"[All Fields] AND "knee"[All Fields]) AND "arthroplasty"[All Fields])) OR "total knee arthroplasty"[All Fields]) OR (((“"arthroplasty, replacement, knee"[MeSH Terms] OR (("arthroplasty"[All Fields] AND "replacement"[All Fields]) AND "knee"[All Fields])) OR "knee replacement arthroplasty"[All Fields]) OR (("total"[All Fields] AND "knee"[All Fields]) AND "replacement"[All Fields])) OR "total knee replacement"[All Fields])) OR (((“arthroplasty, replacement, knee"[MeSH Terms] OR (("arthroplasty"[All Fields] AND "replacement"[All Fields]) AND "knee"[All Fields])) OR "knee replacement arthroplasty"[All Fields]) OR ("knee"[All Fields] AND "arthroplasty"[All Fields])) OR "knee arthroplasty"[All Fields])) OR (((("arthroplasty, replacement, knee"[MeSH Terms] OR (("arthroplasty"[All Fields] AND "replacement"[All Fields]) AND "knee"[All Fields])) "knee replacement arthroplasty"[All Fields]) OR ("knee"[All Fields] AND"replacement"[All Fields])) OR "knee replacement"[All Fields])) OR ("TKA"[Title/ Abstract] OR "TKR"[Title/Abstract])) ((((()"genu valgum"[MeSHTerms] OR ("genu"[All Fields] AND "valgum"[All Fields])) OR "genu valgum"[All Fields]) OR ("genu"[All Fields] AND "valga"[All Fields])) OR "genu valga"[All Fields]) OR (((("genu valgum"[MeSH Terms] OR ("genu"[All Fields] AND "valgum"[All Fields])) OR"genu valgum"[All Fields]) OR ("knock"[All Fields] AND "knee"[All Fields])) OR "knock knee"[All Fields])) OR "knock knees"[Title/Abstract])) ((((()((("arthroplasty, replacement, knee"[MeSH Terms] OR (("arthroplasty"[All Fields] AND "replacement"[All Fields]) AND "knee"[All Fields])) OR "knee replacement arthroplasty"[All Fields]) OR (("total"[All Fields] AND "knee"[All Fields]) AND "arthroplasty"[All Fields])) OR "total knee arthroplasty"[All Fields]) OR (((("arthroplasty, replacement, knee"[MeSH Terms] OR (("arthroplasty"[All Fields] AND "replacement"[All Fields]) AND "knee"[All Fields])) OR "knee replacement arthroplasty"[All Fields]) OR (("total"[All Fields] AND "knee"[All Fields]) AND "replacement"[All Fields])) OR "total knee replacement"[All Fields])) OR (((“"arthroplasty, replacement, knee"[MeSH Terms] OR (("arthroplasty"[All Fields] AND "replacement"[All Fields]) AND "knee"[All Fields])) OR "knee replacement arthroplasty"[All Fields]) OR ("knee"[All Fields] AND "arthroplasty"[All Fields])) OR "knee arthroplasty"[All Fields])) OR (((“"arthroplasty, replacement, knee"[MeSH Terms] OR (("arthroplasty"[All Fields] AND "replacement"[All Fields]) AND "knee"[All Fields])) OR "knee replacement arthroplasty"[All Fields]) OR ("knee"[All Fields] AND "replacement"[All Fields])) OR "knee replacement"[All Fields])) OR ("TKA"[Title/ Abstract] OR

"TKR"[Title/Abstract])) AND "valgus"[All Fields] OR "Joint Deformities, Acquired"[Mesh]) 
Table 2 Inclusion and exclusion criteria

\begin{tabular}{cl}
\hline Inclusion & \\
1 & Valgus deformities $>10$ degrees \\
2 & Intervention: Primary total knee arthroplasty \\
3 & Intervention: peroneal nerve release \\
4 & $\begin{array}{l}\text { Articles reporting soft tissue release procedures and pre-and postopera- } \\
\text { tive clinical outcomes (e.g., measurements of alignments and common } \\
\text { peroneal nerve palsy) }\end{array}$ \\
5 & Prospective or retrospective study design. \\
6 & Articles written in English or Dutch. \\
Exclusion & \\
1 & Valgus deformities $<10$ degrees \\
2 & Genu varus, recurvatum, neutral or mixed alignment populations \\
3 & Previous knee surgery, unicompartimental knee arthroplasties or revisions \\
4 & Double publication of the same cohort \\
5 & Systematic reviews, cadaver studies, case reports or studies $<1980$
\end{tabular}

osteotomies, common peroneal nerve palsy (duration, preoperative valgus alignment), other complications (residual valgus alignment). In case of unavailable or unspecified information, the authors were contacted and asked to provide the missing information.

\section{Methodological quality assessment}

The quality of the non-randomized studies was assessed by the first author (X), utilizing the Methodological Index for Non-Randomized Studies (MINORS) [44]. In case of any doubt, the second reviewer $(\mathrm{X})$ was consulted to determine the quality of the study. The outcome of the index per study is stated in Table 3.

\section{Statistical analysis}

Means and standard deviations were presented and calculated. Reported medians and ranges were transformed into weighted means and estimated standard deviation by the methods of Hozo et al. [45] and Walter et al. [46].

Heterogeneity was assessed using $\mathrm{I}^{2}$ and $X^{2}$-tests, where an $\mathrm{I}^{2}$ of $<25 \%$ is considered low; $25-50 \%$ as moderate; $>50 \%$ as strong and $>75 \%$ as substantially heterogeneous by the methods Higgins et al. [24] In case of substantial heterogeneity between studies $\left(\mathrm{I}^{2}>75 \%\right)$, a qualitative/narrative data extraction was performed [24]. As the heterogeneity of the CPNP incidence was not substantial among studies, data were pooled using a fixedeffect model and weighted on sample size. Because of substantial heterogeneity between perioperative continuous outcomes (alignments), these outcomes were qualitatively/narratively described. Pooled CPNP rates were log-transformed to calculate 95\% Confidence Intervals. Chi2 tests were performed to assess differences between sub-groups. A $p$-value $<0.05$ was considered statistically significant. The Analysis was conducted using $\mathrm{R}$ version 4.0.2 ( $\mathrm{R}$ Foundation statistical computing, Vienna, Austria) with "Metafor package" (Maastricht University, Maastricht, Netherlands).

\section{Results}

Twenty-seven studies were included, representing 1397 valgus knees. Nineteen studies performed only a lateral STR and 8 an OT, including 2 studies with an MFEO and 6 studies LFEO (Table 3). All OT studies used also a lateral STR except one [31]. Two studies performed a PNR in addition to their STR $[8,40]$. There was female predominance (mean $70.4 \%$, range $29-94 \%$ ). The pooled mean age was $67.2 \pm 9.1$ (range 54-74) and the mean BMI ranged from 23 to $30 \mathrm{~kg} / \mathrm{m}^{2}$, but was only reported in 6 studies [8, 17, 30, 33, 36, 47]. Preoperative diagnoses included 746 (59.4\%) patients with OA, 219 (17.5\%) with rheumatoid arthritis, $27(2.2 \%)$ with posttraumatic OA and $263(21.0 \%)$ had an unknown aetiology. Surgery was performed by a medial parapatellar arthrotomy in 16 and a lateral patellar arthrotomy in 11 studies. The weighted mean follow-up was 4.5 years (range, 0.6-10.5).

\section{Quality of the studies}

One randomized controlled trial [33] and 26 non-randomized studies were included. The non-randomized studies consisted of 11 prospective and 15 retrospective cohorts. To estimate the risk of bias of the non-randomized studies, the MINORS criteria were calculated. Two comparative studies had a mean MINOR score of 16.5 (range 16-17) out of 24. The other 24 studies were non-comparative studies with a mean of 10.7 (range, 9-13) out of 16 . Only 3 studies (11.5\%) reported a prospective calculation of the sample size. 


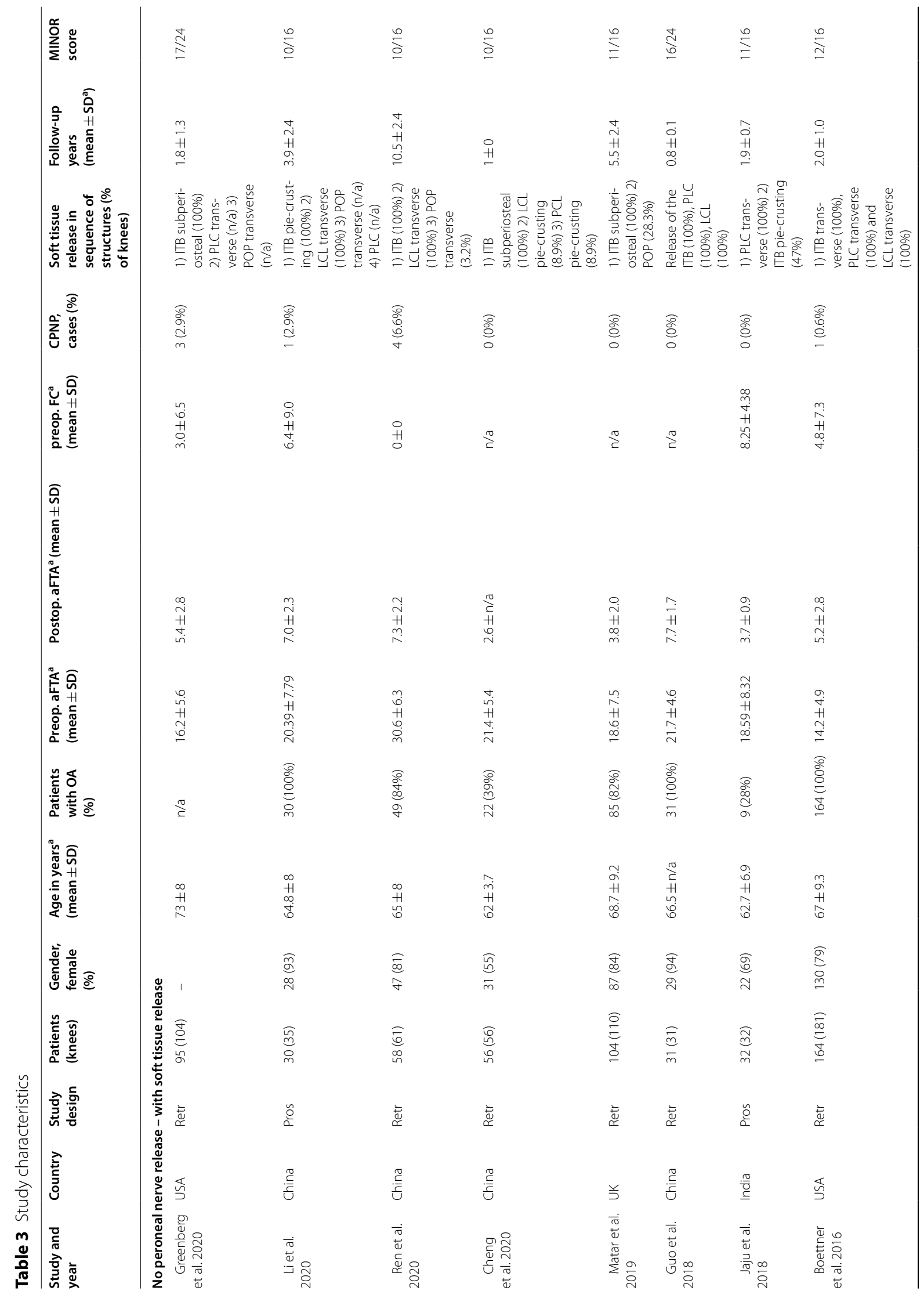


Puijk et al. Journal of Experimental Orthopaedics $\quad$ (2022) 9:12

Page 5 of 12

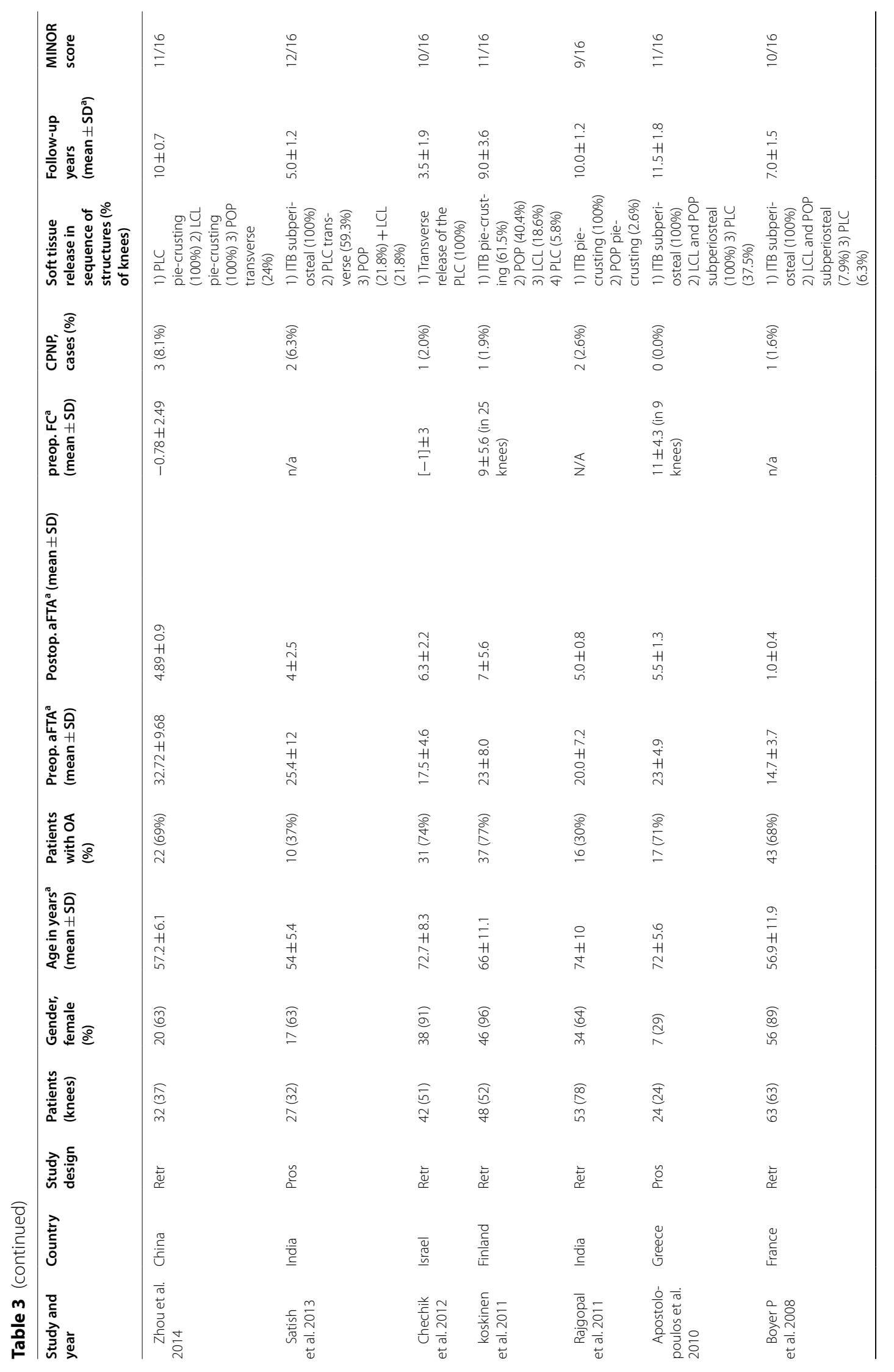


Puijk et al. Journal of Experimental Orthopaedics $\quad$ (2022) 9:12

Page 6 of 12

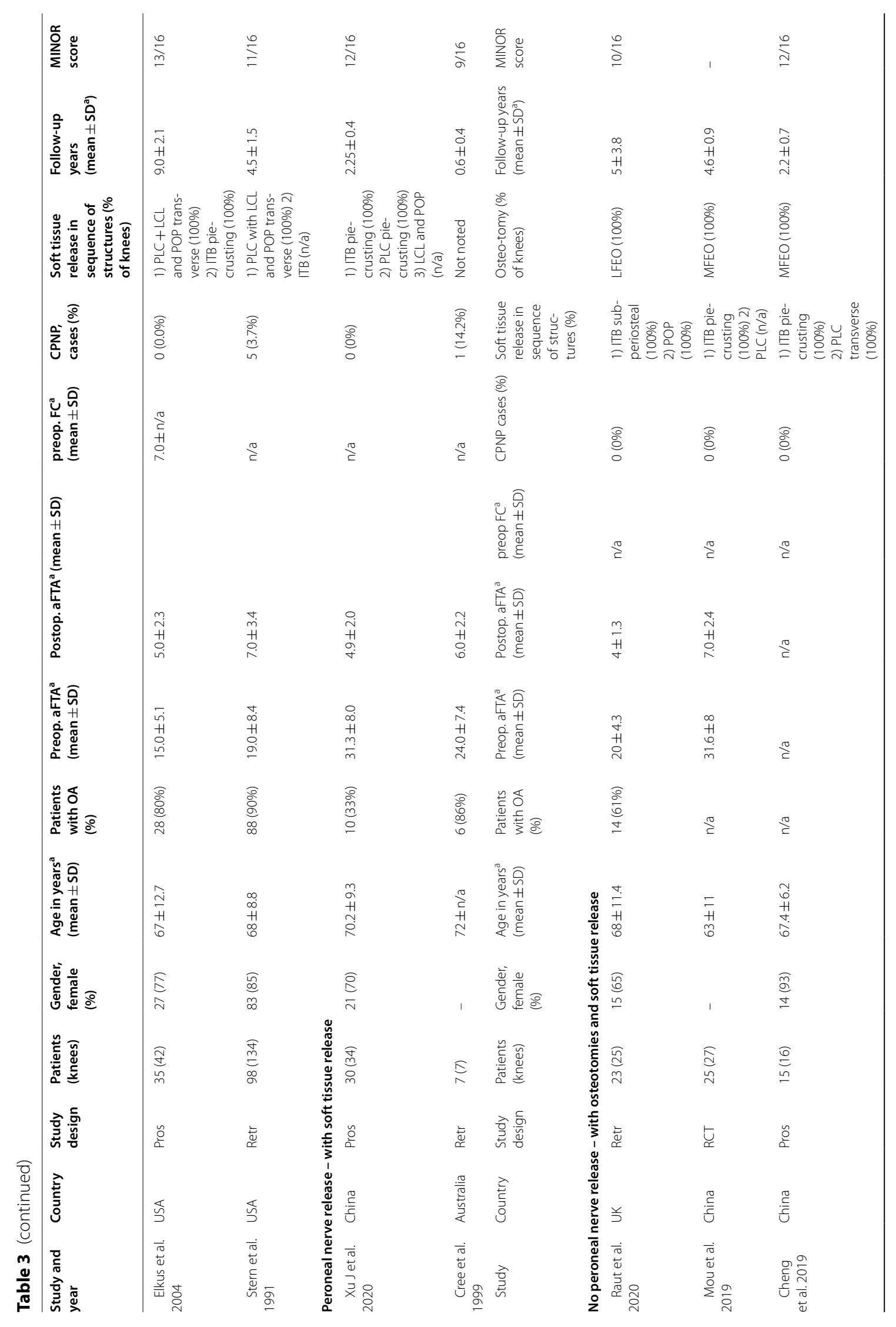




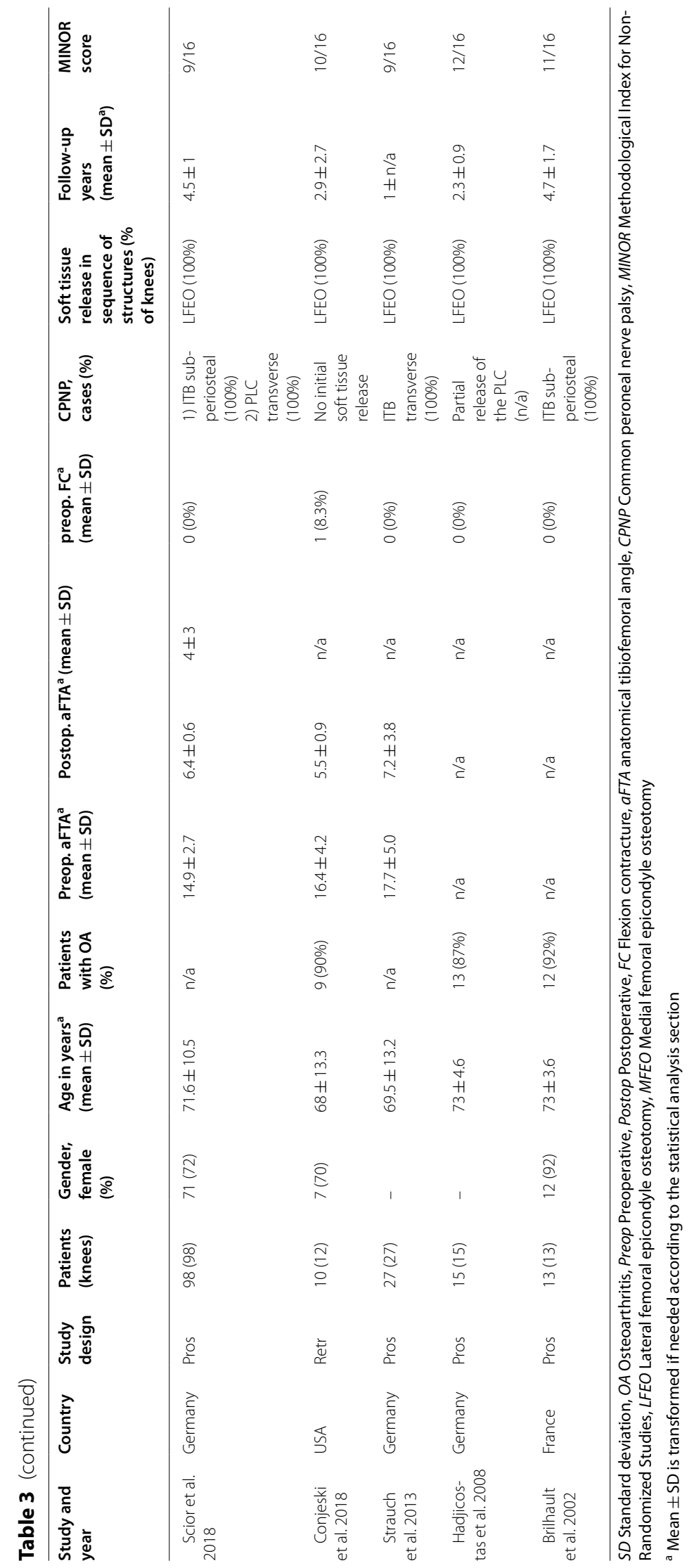




\section{Common peroneal nerve palsy ratio}

In 27 studies, 26 cases of CPNP were reported over a total of 1397 TKA-V (1.9\%) (Table 4). The CPNP ratio was comparable between the studies that performed a PNR in 41 knees and the 17 studies that performed only an STR without PNR in 1123 knees ( $2.4 \%$ vs. $2.1 \%, p=0.90)$. The pooled mean age and female predominance between groups was comparable (70 vs. 67 years and $70 \%$ vs. $73 \%$ ). Also, no significant difference in CPNP rate was found between the studies that performed an STR in 1164 knees and an OT in 233 knees $(2.2 \%$ vs. $0.4 \%, p=0.11)$. The pooled mean aga and female predominance between the STR and OT group were (67 vs. 70 years, $71 \%$ vs. $78 \%)$. The study of Conjeski et al. [31] was responsible for the only CPNP case in the entire OT group $(n=233)$ but was also the single study that used no additional lateral STR and has let the piece of the OT healed in situ without use of internal fixation. Three studies did not explicitly describe CPNP cases in their complication section, therefore it was assumed that CPNP did not occur in those studies [35, 37, 48]. No study clarified if a CPNP case was developed from a patient with posttraumatic OA. Nevertheless, only two studies had patients (11 knees) with posttraumatic OA, where also CPNP cases (7 knees) were reported $[15,16]$. Information on whether these CPNP cases occurred in one of these 11 knees was lacking.

\section{Soft tissue releases}

In the 19 studies that solely performed a lateral STRs, a large variation in released ligaments was present compared with the studies that performed an OT, that mainly released the ITB and PLC. A single study in the OT group performed a POP release [35]. A sub-analysis was performed to approximate the difference in CPNP rate between different specifically released ligaments (Table 5). Between the releases of different ligaments or the manner of those releases (pie-crusting, subperiosteal or transverse), no significant difference in CPNP rate was found. Only studies that reported that all patients underwent a release of a specific ligament were included for sub-analysis. One study was excluded from any analysis due to a lack of data [40].

\section{Pre and postoperative alignments}

The overall weighted mean pre- and postoperative aFTAs was $19.5 \pm 8.4$ and $5.3 \pm 2.7$ degrees (Table 6). The PNR group was the only group with a considerable larger weighted mean preoperative aFTA $(30.1 \pm 1.3)$, and therefore, a larger Valgus Correction Angle (VCA) $\left(25.0^{\circ}\right)$. All weighted postoperative aFTAs were comparable between the different groups. Due to a high heterogeneity between studies $\left(\mathrm{I}^{2}>0.80\right)$, no statistical analysis could be performed. Regarding to the CPNP cases, 4 studies reported the individual preoperative aFTA of 4 CPNP cases, which were $19^{\circ}$ [49], $25^{\circ}$ [47], $26^{\circ}$ [31] and $38^{\circ}$ [40]. Flexion contracture angles were reported in 11 studies (41\%), with an overall weighted mean of $4.1 \pm 4.8^{\circ}$. No individual flexion contractures of CPNP cases were reported in the studies.

\section{Discussion}

In this systematic review, the most important finding was the overall CPNP ratio of $1.9 \%$ after TKA-V. No significant differences in CPNP rate were found between TKA-V with and without PNR (2.4\% vs $2.1 \%)$, between TKA-V with lateral STR $(2.2 \%)$ or with OT $(0.4 \%)$ and between the releases of different ligaments or the manner of those releases (pie-crusting, subperiosteal or transverse).

The obtained overall CPNP ratio of $1.9 \%$ in this study falls within the known range of TKA-V $(0.3 \%-9.5 \%)[3$, 9-17]. Other systematic review reported a range of $0.01 \%$ to $4.3 \%$, like the one of Carender et al. [2] and RodríguezMerchán et al. [50]. Currently, controversy still exists related to valgus deformity being a predisposing factor of CPNP. Studies that have investigated the location of the CPN, indicate that the CPN can be jeopardized by a direct injury due to pie-crusting or a transverse release of the ITB or PLC in well-aligned and valgus knees [19, $20,51]$. However, in our review, we could not confirm the increase of risk to injure the CPN by different ligament releases. Therefore, our results may support the

Table 4 Prevalence of common peroneal nerve palsy

\begin{tabular}{|c|c|c|c|c|c|c|c|}
\hline Treatment group & Studies (n) & Patients (No of knees) & CPNP cases $(\%)$ & $\begin{array}{l}\text { Pooled } \\
\text { proportion } \\
(\%)\end{array}$ & $95 \% \mathrm{Cl}(\%)$ & $\begin{array}{l}\text { Heterogeneity } \\
\mathrm{I}^{2}-(\%)\end{array}$ & $P$-value \\
\hline Osteotomy & 8 & $226(233)$ & $1(0.4 \%)$ & 0.43 & $0.01-2.37$ & $0 \%$ & \\
\hline Soft tissue release & 19 & 1029 (1164) & $25(2.2 \%)$ & 2.15 & $1.39-3.15$ & $0 \%$ & 0.11 \\
\hline PNR & 2 & $37(41)$ & $1(2.4 \%)$ & 2.44 & $0.06-12.86$ & $46 \%$ & \\
\hline No PNR & 17 & $992(1123)$ & $24(2.1 \%)$ & 2.14 & $1.37-3.16$ & $2 \%$ & 0.90 \\
\hline Overall & 27 & 1255 (1397) & $26(1.9 \%)$ & 1.86 & $1.22-2.72$ & $0 \%$ & \\
\hline
\end{tabular}

No Number of, CPNP Common peroneal nerve palsy, PNR Peroneal nerve release, CI Confidence interval 
Table 5 Specific ligament release and CPNP ratio

\begin{tabular}{llll}
\hline Released ligament & A Studies $(\mathbf{n})$ & Treated knees (\%) & CPNP cases, (\%) \\
\hline No ITB releases & 4 & $114(9.7 \%)$ & $4(3.5 \%)$ \\
ITB release overall & 19 & $1057(90.3 \%)$ & $14(1.3 \%)$ \\
$\quad$ Pie-crusting & 6 & $232(21.9 \%)$ & $3(1.3 \%)$ \\
Subperiosteal & 9 & $525(49.7 \%)$ & $6(1.1 \%)$ \\
Transverse & 2 & $208(19.7 \%)$ & $1(0.5 \%)$ \\
Unclear ${ }^{A}$ & 2 & $92(8.7 \%)$ & $0(0.0 \%)$ \\
No PLC releases & 7 & $326(33.2 \%)$ & $7(2.1 \%)$ \\
PLC release overall & 10 & $656(66.8 \%)$ & $10(1.5 \%)$ \\
Pie-crusting & 2 & $71(10.8 \%)$ & $3(2.4 \%)$ \\
Transverse & 7 & $553(84.3 \%)$ & $7(1.3 \%)$ \\
Unclear ${ }^{A}$ & 1 & $32(4.9 \%)$ & $0(0.0 \%)$ \\
No LCL releases & 18 & $608(52.7 \%)$ & $7(1.2 \%)$ \\
LCL release overall & 8 & $545(47.3 \%)$ & $14(2.6 \%)$ \\
No POP releases & 14 & $700(75.7 \%)$ & $9(1.3 \%)$ \\
POP release overall & 4 & $225(24.3 \%)$ & $5(2.2 \%)$ \\
\hline
\end{tabular}

CPNP Common peroneal nerve palsy, ITB Iliotibial band, PLC Posterior lateral capsule, LCL Lateral collateral ligament, POP Popliteus tendon. A: Studies that released a lateral soft tissue, but without specifying in which manner this was performed

Studies that performed a specific ligament release on only a part of the total study population were excluded for analysis. One study [40] was excluded for analysis due to lack of data

Table 6 Pre and -postoperative alignments

\begin{tabular}{|c|c|c|c|c|c|}
\hline & Studies $^{A}$ & Patients (No of knees) & $\begin{array}{l}\text { Mean preoperative } \\
\mathrm{aFTA}^{\circ} \pm \mathrm{SD}\end{array}$ & $\begin{array}{l}\text { Mean Postoperative } \\
\mathrm{aFTA}^{\circ} \pm \text { SD }\end{array}$ & mean VCA ${ }^{\mathrm{B}}$ \\
\hline Osteotomy & 5 & $189(190)$ & $18.5 \pm 5.7$ & $6.2 \pm 0.7$ & 12.3 \\
\hline Soft tissue releases & 19 & $1029(1164)$ & $18.6 \pm 6.2$ & $5.2 \pm 2.5$ & 13.4 \\
\hline PNR & 2 & $37(41)$ & $30.1 \pm 1.3$ & $5.1 \pm 0.3$ & 25.0 \\
\hline No PNR & 17 & $992(1123)$ & $19.3 \pm 6.1$ & $5.2 \pm 2.5$ & 14.1 \\
\hline Overall & 24 & $1212(1353)$ & $19.5 \pm 8.4$ & $5.3 \pm 2.7$ & 14.2 \\
\hline
\end{tabular}

CPNP Common peroneal nerve palsy, VCA Valgus correction angle, aFTA Anatomical femorotibial angle

No statistical analysis is performed due to a substantial heterogeneity between the studies. All studies are weighted by the number of operated knees. Three study were excluded due to lack of data $[29,30,32]$. Valgus correction angle is calculated by postoperative aFTA minus preoperative aFTA

theory that most CPNPs probably occur due to postoperative mechanical damage, like traction and compression, instead of a direct injury. Besides, one large registrybased study by Christ et al. [3], including 383,060 primary TKA procedures, found that preoperative valgus alignments increase the risk of developing a CPNP significantly (OR 4.19). Also, Idusuyi et al. [4], found a relative risk of CPNP 12 times greater for patients with a $12^{\circ}$ or more valgus deformity. Both studies did not find an association between CPNP and flexion contractures. However, according to Christ et al. [3], this may be because the diagnosis code for flexion contractures is not consistently noted as that of valgus deformities in their registry. Therefore, the data may be biased. Other studies, like Park et al. [12] and Schinsky et al. [52] found an overall incidence of $0.53 \%$ and $1.3 \%$ but did not find any relation between valgus deformities and CPNP. However, all these studies used mixed preoperative alignments. Therefore, it is difficult to compare the CPNP ratio of this systematic review, with the incidence of other reviews or studies. Eventually, knowing that larger previous studies showed an increase in CPNP incidence in valgus knees, we would advise clinicians to perform a TKA-V with extra care. This would enable PNR as an option for severe valgus deformities since the procedure is minimally invasive and may lead to preventing CPNP. However, this current review did not find a significant difference in CPNP incidence between the studies that performed a TKA-V with and without PNR. Regarding the 2 studies utilizing a PNR, the study of Cree et al. [40] is a small retrospective study and the recent study of $\mathrm{Xu}$ et al. [8] is a small prospective study, both studies performed the same surgical 
technique. The studies together account for a population of 41 knees in which 1 developed a CPNP. Focussing on that single CPNP case, the study of Cree et al. [40] mentioned that the CPN remained too tight after an extensive PNR due to a vast preoperative aFTA of $38^{\circ}$ and VCA of $30^{\circ}$. Therefore, this CPNP developed assumably due to the postoperative stretch of the CPN. Furthermore, comparing the perioperative alignments of the 2 PNR studies with the non-PNR studies show that the preoperative aFTA of the 2 PNR studies is substantially bigger $\left(30.1^{\circ}\right.$. vs $19.3^{\circ}$ ). However, it is difficult to compare these alignments without any statistical analysis due to the high heterogeneity between the studies. It is noticeable that the 4 individual CPNP cases that are mentioned in the 4 studies, all have a higher preoperative aFTA $\left(19^{\circ}[49], 25^{\circ}\right.$ [47], $26^{\circ}$ [31] and $38^{\circ}$ [40]) than the overall mean aFTA of all the studies in this review.

In the end, the results in this review suggest that a PNR procedure is not effective. However, it is difficult to assume such an interpretation because only two small sample sized studies were found that used a PNR prior to TKA and met the inclusion criteria of our systematic review $[8,40]$. Future research should further investigate PNR in larger study populations and preferably with a comparison group, which would make it easier to interpret results.

Like all studies, some limitations need to be discussed. Firstly, the considerable heterogeneity between the included studies, possibly caused by our caution to minimize selection bias in including studies for this review. However, due to the low incidence of CPNP and the focus on valgus deformities, a comprehensive literature search was needed. Secondly, the review lacks important detailed information about the individual cases who developed a CPNP in the studies. In addition, preoperative data of the knees, like knee extension angles and stress radiographs to assess whether there is a fixed valgus deformity are missing in most studies. Therefore, it is important for future studies to specify the manner and degree of the surgeries and to comprehensively note the pre and postoperative data of the knees. Thirdly, the scarce of studies investigating a PNR is an insurmountable problem, which made it impossible to draw conclusions. However, this review provides a basis for future work investigating PNR in valgus knees to prevent CPNP.

\section{Conclusion}

To our knowledge, this is the first systematic review that provides insight into the current literature about preventing CPNP with a PNR after TKA-V. An overall CPNP ratio of $1.9 \%$ in valgus knees after TKA was found. There was no direct evidence that using a PNR would be more effective than not using a PNR in preventing a CPNP. However, it was impossible to draw conclusions, due to the scarce amount of literature. Therefore, larger studies comparing TKA-V with and without PNR are needed to appropriately define the efficiency of a PNR. This systematic review is the first step in this regard.

\begin{abstract}
Abbreviations
TKA: Total Knee Arthroplasty; TKA-V: Total Knee Arthroplasty in Valgus deformities of $>10^{\circ}$ aFTA; STR: Soft Tissue Release; OT: Osteotomy; ITB: lliotibial Band; LCL: Lateral Collateral Ligament; PLC: Posterolateral Capsule; POP: Popliteus tendon; CPNP: Common Peroneal Nerve Palsy; CPN: Common Peroneal Nerve; PNR: Peroneal Nerve Release; aFTA: anatomic FemoroTibial Angle;VCA: Valgus Correction Angle; LFEO: Lateral Femoral Epicondyle Osteotomy; MFEO: Medial Femoral Epicondyle Osteotomy.
\end{abstract}

\section{Supplementary Information}

The online version contains supplementary material available at https://doi. org/10.1186/s40634-021-00443-x.

Additional file 1. Figure S1. PRISMA flow diagram. The flow diagram of study selection per guidelines from the Preferred Reporting Items for Systematic Reviews and Meta-Analyses (PRISMA) group.

\section{Acknowledgements}

We would like to thank Marjolein Schager of the Spaarne Gasthuis for assistance in drafting the manuscript and Willy Salemink of the Spaarne Gasthuis Library for the librarian-assisted search.

\section{Authors' contributions}

RP performed the literature search, scanned all abstracts and full texts of the included articles, determined the quality of the studies and wrote the manuscript. RR and LK screened all abstracts and full texts as a second author and helped to draft the manuscript. IS provided suggestions on the review process, statistical analyses and manuscript; and checked the data extraction. PN coordinated this study and participated in its design. All authors read and approved the final manuscript.

\section{Funding}

This research did not receive any specific grant from funding agencies in the public, commercial, or not-for-profit sectors.

\section{Declarations}

Ethics approval and consent to participate

No ethical approval was necessary for this study.

No informed consent was necessary for this study.

\section{Competing interests}

None.

\section{Author details}

${ }^{1}$ Department of Orthopaedic Surgery, Spaarne Gasthuis, Spaarnepoort 1, 2130AT, Hoofddorp, the Netherlands. ${ }^{2}$ Specialized Center of Orthopedic Research and Education (SCORE), Xpert Orthopedie, Amsterdam, the Netherlands.

Received: 2 November 2021 Accepted: 8 December 2021

Published online: 20 January 2022 


\section{References}

1. Asp JP, Rand JA (1990) Peroneal nerve palsy after total knee arthroplasty. Clin Orthop Relat Res (261):233-237.

2. Carender CN, Bedard NA, An Q, Brown TS (2020) Common peroneal nerve injury and recovery after total knee arthroplasty: a systematic review. Arthroplast Today 6(4):662-667

3. Christ AB, Chiu YF, Joseph A, Westrich GH, Lyman S (2019) Incidence and risk factors for peripheral nerve injury after 383,000 Total knee arthroplasties using a New York state database (SPARCS). J Arthroplast 34:2473-2478

4. Idusuyi OB, Morrey BF (1996) Peroneal nerve palsy after total knee arthroplasty. Assessment of predisposing and prognostic factors. J Bone Joint Surg Am 78:177-184

5. Poage C, Roth C, Scott B (2016) Peroneal nerve palsy: evaluation and management. J Am Acad Orthop Surg 24:1-10

6. Görtz S, Guilherme C, Gracitelli, Bugbee WD (2017) 27 - Valgus Malalignment: Diagnosis, Osteotomy Techniques, and Clinical Outcomes. In: Noyes' Knee Disorders: Surgery, Rehabilitation, Clinical Outcomes (Second Edition). Elsevier, p 848-857. https://doi.org/10.1016/B978-0-323-32903-3. 00027-5.

7. Ranawat AS, Ranawat CS, Elkus M, Rasquinha VJ, Rossi R, Babhulkar S (2005) Total knee arthroplasty for severe valgus deformity. J Bone Joint Surg Am 87(Suppl 1):271-284

8. Xu J, Liu H, Luo F, Lin Y (2020) Common peroneal nerve 'pre-release' in total knee arthroplasty for severe valgus deformities. Knee 27:980-986

9. Koskinen E, Remes V, Paavolainen P, Harilainen A, Sandelin J, Tallroth K et al (2011) Results of total knee replacement with a cruciate-retaining model for severe valgus deformity-a study of 48 patients followed for an average of 9 years. Knee 18:145-150

10. Matar HE, Thangaraj R, Saraogi A, Raut V (2019) High medium-term survivorship of cruciate-retaining Total knee arthroplasties (110 knees) for Valgus deformity. J Knee Surg 34(4):422-426

11. Nercessian OA, Ugwonali OF, Park S (2005) Peroneal nerve palsy after total knee arthroplasty. J Arthroplast 20:1068-1073

12. Park JH, Restrepo C, Norton R, Mandel S, Sharkey PF, Parvizi J (2013) Common peroneal nerve palsy following total knee arthroplasty: prognostic factors and course of recovery. J Arthroplast 28:1538-1542

13. Ren J, Zhang X, Wulamu W, Yushan N, Aaimaiti A, Cao L (2020) Total knee arthroplasty with the least-constrained implant possible for type II valgus knee> 20: a 3-14 years' follow-up. Arthroplasty 2:1-7

14. Rossi R, Rosso F, Cottino U, Dettoni F, Bonasia DE, Bruzzone M (2014) Total knee arthroplasty in the valgus knee. Int Orthop 38:273-283

15. Satish BR, Ganesan JC, Chandran P, Basanagoudar PL, Balachandar D (2013) Lateral parapatellar approach without tibial tubercle osteotomy for fixed valgus deformity correction in total knee arthroplasty. JBJS Essent Surg Tech 3(4): e20

16. Stern SH, Moeckel BH, Insall JN (1991) Total knee arthroplasty in valgus knees. Clin Orthop Relat Res (273):5-8

17. Zhou X, Wang M, Liu C, Zhang L, Zhou Y (2014) Total knee arthroplasty for severe valgus knee deformity. Chin Med J (Engl) 127:1062-1066

18. Aglietti P, Lup D, Cuomo P, Baldini A, De Luca L (2007) Total knee arthroplasty using a pie-crusting technique for valgus deformity. Clin Orthop Relat Res 464:73-77

19. Bruzzone M, Ranawat A, Castoldi F, Dettoni F, Rossi P, Rossi R (2010) The risk of direct peroneal nerve injury using the Ranawat "inside-out" lateral release technique in valgus total knee arthroplasty. J Arthroplast 25:161-165

20. Clarke HD, Fuchs R, Scuderi GR, Scott WN, Insall JN (2005) Clinical results in valgus total knee arthroplasty with the "pie crust" technique of lateral soft tissue releases. J Arthroplast 20:1010-1014

21. Lange J, Haas S (2017) Correcting severe valgus deformity: taking out the knock. Bone Joint J 99:60-64

22. Cheng W, Li Z, Zhang J, Cao Q, Yu H, Qi L et al (2021) A lateral parapatellar approach with iliotibial band dissection from the Gerdy tubercle for total knee arthroplasty of the valgus knee. Exp Ther Med 21:38

23. Elkus M, Ranawat CS, Rasquinha VJ, Babhulkar S, Rossi R, Ranawat AS (2004) Total knee arthroplasty for severe valgus deformity: five to fourteen-year follow-up. JBJS 86:2671-2676

24. Li H, Ponzio DY, Ong A, Wei W, Wang B, Li Z et al (2020) Total knee arthroplasty for fixed valgus deformity correction using a modified lateral parapatellar approach. J Knee Surg 33:372-377
25. Rajgopal A, Dahiya V, Vasdev A, Kochhar H, Tyagi V (2011) Long-term results of total knee arthroplasty for valgus knees: soft-tissue release technique and implant selection. J Orthop Surg 19:60-63

26. Apostolopoulos AP, Nikolopoulos DD, Polyzois I, Nakos A, Liarokapis S, Stefanakis G et al (2010) Total knee arthroplasty in severe valgus deformity: interest of combining a lateral approach with a tibial tubercle osteotomy. Orthop Traumatol Surg Res 96:777-784

27. Greenberg A, Kandel L, Liebergall M, Mattan Y, Rivkin G (2020) Total knee arthroplasty for Valgus deformity via a lateral approach: clinical results, comparison to medial approach, and review of recent literature. J Arthroplast 35:2076-2083

28. Whiteside LA (1999) Selective ligament release in total knee arthroplasty of the knee in valgus. Clin Orthop Relat Res 367:130-140

29. Brilhault J, Lautman S, Favard L, Burdin P (2002) Lateral femoral sliding osteotomy: lateral release in total knee arthroplasty for a fixed valgus deformity. J Bone Joint Surg Br 84:1131-1137

30. Cheng X, Wang Z, Zhang Y, Wang M, Zhang X (2019) Tightening medial collateral ligament during total knee arthroplasty for patients with fixed valgus deformity: a novel technique. J Orthop Surg (Hong Kong) 27:2309499019834695

31. Conjeski JM, Scuderi GR (2018) Lateral femoral Epicondylar osteotomy for correction of fixed Valgus deformity in Total knee arthroplasty: a technical note. J Arthroplast 33:386-390

32. Hadjicostas PT, Soucacos PN, Thielemann FW (2008) Computer-assisted osteotomy of the lateral femoral condyle with non-constrained total knee replacement in severe valgus knees. J Bone Joint Surg Br 90:1441-1445

33. Mou P, Zeng Y, Pei F, Zhou Z, Shen B, Kang P et al (2019) Medial femoral epicondyle upsliding osteotomy with posterior stabilized arthroplasty provided good clinical outcomes such as constrained arthroplasty in primary total knee arthroplasty with severe valgus deformity. Knee Surg Sports Traumatol Arthrosc 27:2266-2275

34. Mullaji AB, Shetty GM (2010) Lateral epicondylar osteotomy using computer navigation in total knee arthroplasty for rigid valgus deformities. $J$ Arthroplast 25:166-169

35. Raut V, Matar HE, Singh A (2020) Satisfactory medium-term outcomes with lateral condylar sliver osteotomy to correct valgus deformity in total knee replacements. Knee Surg Sports Traumatol Arthrosc 28:1394-1399

36. Scior W, Hilber F, Hofstetter M, Graichen H (2018) Short-term and mid term results of lateral condyle sliding osteotomy in the treatment of valgus total knee arthroplasty: a successful therapy option in grade 2 valgus total knee arthroplasty. Knee 25:466-472

37. Strauch M, von Eisenhart RR, Graichen H (2013) A new navigation-based technique for lateral distalizing condylar osteotomy in patients undergoing total knee arthroplasty with fixed valgus deformity. Knee Surg Sports Traumatol Arthrosc 21:2263-2270

38. Ang CL, Fook S, Chia SL, Chin PL, Lo NN, Yeo SJ (2014) Unconstrained arthroplasty in type II valgus knees: posterior stabilized or cruciate retaining? Knee Surg Sports Traumatol Arthrosc 22:666-673

39. Easley ME, Insall JN, Scuderi GR, Bullek DD (2000) Primary constrained condylar knee arthroplasty for the arthritic valgus knee. Clin orthop Relat Res 380(2):58-64

40. Cree AK, Coolican MR, Tonkin MA (1998) Prevention of common peroneal nerve palsy after surgery for valgus deformity about the knee. Knee 5:261-265

41. Liberati A, Altman DG, Tetzlaff J, Mulrow C, Gøtzsche PC, loannidis JP et al (2009) The PRISMA statement for reporting systematic reviews and metaanalyses of studies that evaluate healthcare interventions: explanation and elaboration. BMJ 339:b2700

42. Ouzzani M, Hammady H, Fedorowicz Z, Elmagarmid A. Rayyan - a web and mobile app for systematic reviews. Systematic Reviews (2016) 5:210. https://doi.org/10.1186/s13643-016-0384-4.

43. Prill R, Karlsson J, Ayeni OR, Becker R (2021) Author guidelines for conducting systematic reviews and meta-analyses. Knee Surg Sports Traumatol Arthrosc 29:2739-2744

44. Slim K, Nini E, Forestier D, Kwiatkowski F, Panis Y, Chipponi J (2003) Methodological index for non-randomized studies (minors): development and validation of a new instrument. ANZ J Surg 73:712-716

45. Hozo SP, Djulbegovic B, Hozo I (2005) Estimating the mean and variance from the median, range, and the size of a sample. BMC Med Res Methodol 5:13 
46. Walter SD, Yao X (2007) Effect sizes can be calculated for studies reporting ranges for outcome variables in systematic reviews. J Clin Epidemiol 60:849-852

47. Boettner F, Renner L, Arana Narbarte D, Egidy C, Faschingbauer M (2016) Total knee arthroplasty for valgus osteoarthritis: the results of a standardized soft-tissue release technique. Knee Surg Sports Traumatol Arthrosc 24:2525-2531

48. Guo C, Liu J, Niu D, Ma J, Kou B, Zhang H et al (2018) Clinical application of different operative approach of total knee replacement in knee valgus patients. Retrospective cohort study. Int J Surg 49:80-83

49. Chechik O, Mayer C, Drexler M, Sternheim A, Snir N, Dekel S (2012) Posterolateral capsular release for correction of valgus deformity. J Knee Surg 25:355-360

50. Rodríguez-Merchán EC, De la Corte-Rodríguez H, Encinas-Ullán CA (2020) Peroneal nerve palsy after Total knee arthroplasty: prevalence, risk factors, diagnosis and management. In: Rodríguez-Merchán EC, Gómez-Cardero $P$ (eds) Comprehensive treatment of knee osteoarthritis: recent advances. Springer International Publishing, Cham, pp 135-142

51. Yang D, Shao H, Zhou Y, Tang H, Guo S (2017) Location of the common peroneal nerve in Valgus knees-is the reported safe zone for well-aligned knees applicable? J Arthroplast 32:3539-3543

52. Schinsky MF, Macaulay W, Parks ML, Kiernan H, Nercessian OA (2001)

Nerve injury after primary total knee arthroplasty. J Arthroplast 16:1048-1054

\section{Publisher's Note}

Springer Nature remains neutral with regard to jurisdictional claims in published maps and institutional affiliations.

\section{Submit your manuscript to a SpringerOpen ${ }^{\circ}$ journal and benefit from:}

- Convenient online submission

- Rigorous peer review

- Open access: articles freely available online

- High visibility within the field

- Retaining the copyright to your article

Submit your next manuscript at $\boldsymbol{\nabla}$ springeropen.com 